\title{
Manejo forestal con bases científicas
}

\author{
Klaus von Gadow ${ }^{1}$ \\ Sofía Sánchez Orois ${ }^{1}$ \\ Oscar Alberto Aguirre Calderón²
}

\begin{abstract}
RESUMEN
Los bosques son complejos sistemas abiertos con múltiples funciones. Para mantener altos estándares y la credibilidad de la opinión pública, los responsables del manejo de recursos forestales requieren integrar la experiencia de diferentes disciplinas científicas, incluyendo las humanidades, la física, la ingeniería y las ciencias biológicas, no necesariamente en ese orden. Con frecuencia se postula que el manejo forestal debe ser sustentable, basarse en resultados de investigación validados, ser acorde con estándares ambientales aceptables y ser transparente para la opinión pública; estos objetivos pueden lograrse si: a) se evalúa una variedad de opciones forestales, b) se demuestra el manejo de manera comprensible en el campo y c) se revisan y evalúan continuamente las actividades de manejo considerando diferentes condiciones ecológicas. Con base en estas premisas un concepto práctico para el manejo de un paisaje forestal incluye tres elementos: Diseño de Opciones, Parcelas de Demostración y Análisis de Eventos. El objetivo de este trabajo es presentar un sistema para el manejo de paisajes forestales sobre bases científicas, que incluye los tres elementos anteriores.
\end{abstract}

PALABRAS CLAVE:

Análisis del evento silvícola, diseño de opciones, investigación y demostración.

\begin{abstract}
Forests are complex open systems with multiple functions. To maintain high standards and credibility among the public, forest managers need to draw on the expertise of a variety of scientific disciplines, including the humanities, the physical, the engineering and biological sciences, not necessarily in that order. It is often postulated that forest management should be sustainable, based on validated research results, conform to acceptable environmental standards and be transparent to the public. These objectives can be met a) if various silvicultural options are compared using validated research results, b) if management practices are understandably demonstrated in the field, and c) if management activities are effectively monitored, considering a variety of ecological conditions. Based on these premises, a practical framework for the management of a forested landscape includes three elements: Forest Options Design, Research and Demonstration and Harvest Event Analysis. The objective of the paper is to present a framework for science-based management of a forested landscape, which includes these three elements.
\end{abstract}

KEYWORDS:

Harvest event analysis, forest options design, research and demonstration. 


\section{INTRODUCCIÓN}

En muchas regiones del mundo se acepta que el manejo forestal constituye un gran apoyo para la conservación de los diversos ecosistemas forestales y para mejorar la productividad económica de los bosques. Desde hace doscientos años se establece y desarrolla el principio del manejo forestal sustentable, plasmándose hasta ahora bajo dos filosofías opuestas: el manejo del bosque regular y el manejo de bosque continuo (Thomasius y Schmidt, 1996). En el manejo regular el bosque se cosecha periódicamente y se renueva mediante plantación o regeneración natural dando lugar a un bosque coetáneo; el sistema se caracteriza por un turno de cosecha (rotación) y un crecimiento medio. Por el contrario, en el manejo irregular, los árboles individuales tienen una edad, pero no el bosque en su conjunto, no hay turno de cosecha, ni tampoco un crecimiento promedio (Garfitt, 1995; Gadow y Pummalainen, 2000).

Durante los últimos cien años tuvo lugar en Europa un manejo irregular, hoy en día este sistema de manejo se aplica prácticamente en la mayoría de los bosques en Alemania; no se aplican matarrasas, se prefieren especies arbóreas adecuadas al sitio y se fomenta la regeneración natural. La consecuencia práctica de estas acciones es el desarrollo a gran escala de bosques mixtos e irregulares con estructuras complejas. Dada la complejidad de las estructuras y la gran cantidad de opciones silvícolas posibles, se tiene ahora la obligación de establecer nuevos métodos de manejo forestal; específicamente métodos de inventario, de planificación y de control. El manejo irregular tiene una larga tradición, pero aprovechamientos específicos basados en este sistema se encuentran sólo en ciertos bosques tropicales, en algunos bosques de Norteamérica y en los bosques de selección en Europa. Normalmente se prescribe una estructura ideal tendiente a conformar bosques regulares bajo el esquema del modelo del bosque normal; tal prescripción tiene la forma, por ejemplo, de una curva guía. Meyer (1930) propuso una función para describir la distribución ideal; conceptos más recientes para determinar distribuciones normales se incluyen en los trabajos de Buongiorno y Gilles (2002) y Cancino y Gadow (2001). Estos métodos no tienen una aplicación general, sino que sólo son válidos para un tipo de bosque específico. Otro método implica el uso del área basal normal; siendo el área basal extraída la que excede la normalidad. La utilización de las curvas guías y del área basal normal es muy limitada, frecuentemente las prescripciones rígidas son valoradas negativamente.

Uno de los problemas del manejo forestal es el hecho de que las diferentes opciones silvícolas no son evaluadas. Dado que existe una gran diversidad de opciones, una tarea de la planificación forestal es desarrollar y evaluar estrategias de manejo. La comparación de opciones se realiza para un periodo específico, dentro de un marco temporal. Particularmente en bosques nativos existe normalmente una gran cantidad de posibilidades de manejo, un plan puede ser óptimo para un rodal individual, pero esto no significa necesariamente que sea óptimo para un paisaje en su conjunto.

Además de los métodos de inventario y planificación, el control es una tarea importante en el manejo de bosques nativos. En este marco se puede recomendar un método de control denominado análisis de aclareo, el cual implica una inventarización de los cambios provocados por la intervención. El análisis de aclareo implica un inventario que tiene lugar después del marqueo y antes de la corta, de manera que se puedan analizar los efectos ocasionados por la interven- 
ción, sobre todo los cambios de densidad, de diversidad y del valor de los árboles. La modificación del bosque provocada por la intervención se observa de manera continua por el responsable del manejo forestal, de tal forma que es posible realizar correcciones antes de que la intervención tenga lugar.

\section{Bosques Nativos}

Aproximadamente un tercio de la superficie terrestre está cubierta por bosques. En su enciclopedia Folch y Camarasa (2000) describen 7 tipos de ecosistemas naturales arbolados:

- El bosque tropical húmedo en regiones africanas, americanas, asiáticas e indopacíficas.

- Los bosques templados húmedos en las costas occidentales de América del Norte y América del Sur.

- Los bosques monzónicos en el Sureste de Asia, Australia, Madagascar, América Central y el Caribe, que se caracterizan por la alternancia de un periodo lluvioso y un periodo seco.

- Las sabanas forestales, que incluyen el Cerrado, los Llanos, el Pantanal y el Chaco de America del Sur y los bosques Miombo, la sabana herbácea y arbustiva de África. Estos ecosistemas se caracterizan por una limitada disponibilidad de nutrientes y agua y por los intermitentes incendios en la cubierta arbustiva.

- Los bosques mediterráneos se localizan en áreas pequeñas y fragmentadas en la cuenca del Mediterráneo, en California, en el centro de Chile, en el suroeste de África y en el Sur y Suroeste de Australia.

- El bioma de los bosques caducifolios se localiza en las regiones occidentales de América del Norte, el Este de Asia y en la mayor parte de Europa, extendiéndose en un estrecho cinturón a través de Europa occidental hasta las mesetas de los Urales.

- El bioma del bosque boreal incluye las coníferas de la taiga en Eurasia y Norteamérica y también se extiende por las montañas de Europa central y occidental, el Cáucaso, Asia central, El Himalaya, China y las Montañas Rocallosas en los EUA.

A causa de la imprecisión en los límites entre los diferentes biomas no se puede determinar exactamente su extensión. Esto es evidente en el caso de los bosques caducifolios que limitan con el bosque boreal, el bosque templado húmedo y los sistemas mediterráneos.

Muchos países tropicales enfrentan el grave problema de la degradación del suelo. Durante el quinquenio 1990-1995, Sudamérica perdió 24 millones de ha de bosque, África 19 y Asia 17 (FAO, 2001). Las prioridades del uso forestal varían en función del ingreso per cápita y de la cubierta forestal (Tabla 1).

Los principales factores causantes de la deforestación en muchas regiones del mundo son, generalmente, una tasa de extracción de madera no sustentable, los pastizales y los cultivos anuales y la conversión de bosques naturales en plantaciones de caucho, aceite de palma de cacao y caña de azúcar (Dada, 2000; Nik et al., 2000; Abdul y Appanah, 2000). Los beneficios económicos obtenidos de las operaciones de corta pueden contrarrestarse por los daños ambientales causados a largo plazo y los costos ocasionados a la sociedad. Los sistemas agroforestales y silvopastoriles y la plantación de cultivos anuales entre pequeños rodales son muy comunes en las regiones tropicales, especialmente en América Central (De Camino, 2000; Beer et al., 2000). 
Tabla 1. Prioridades del uso forestal en función del ingreso y la cubierta forestal per cápita. (Emborg y Larsen, 1999)

\begin{tabular}{|l|l|l|l|}
\hline \multirow{2}{*}{$\begin{array}{l}\text { Ingreso } \\
\text { per cápita }\end{array}$} & elevado & Cuestiones ambientales & Desarrollo sustentable \\
\cline { 2 - 4 } & bajo & $\begin{array}{l}\text { Cuestiones de } \\
\text { subsistencia }\end{array}$ & Desarrollo económico \\
\hline \multirow{2}{*}{ baja } & elevada \\
\cline { 2 - 3 } & \multicolumn{2}{|c|}{ Cubierta forestal per cápita } \\
\end{tabular}

Uno de los pilares de la teoría económica es la "tragedia de los comunes". Hardin (1968) argumenta que un recurso común está condenado a desaparecer cuando hay libre acceso al mismo, Bousquet et al. (2000) pudieron verificar los patrones típicos del agotamiento del bosque que apoyan esta teoría. Este problema se puede solucionar mediante la privatización o controlando el acceso al recurso y mediante mecanismos reguladores o incentivos. Los bosques remanentes aislados desempeñan un papel crítico en la conservación de la biodiversidad dentro del paisaje (Harvey et al., 2000), la pregunta es: ¿cuánto tiempo podrán sobrevivir estos bosques remanentes aislados? ¿son los sistemas agroforestales que dependen de los árboles del monte alto realmente sustentables? Para explorar las consecuencias de los escenarios alternativos del uso del suelo se están desarrollando nuevos paquetes de cómputo como el software de simulación multiagente MAS (Bousquet et al., 2000) y el sistema FLORES (Vanclay et al., 2000).

\section{Bosques Manejados}

Es evidente que muchos bosques no se encuentran en estado natural y se carece de un análisis histórico completo de las acciones de manejo aplicadas. Utilizando una clasificación simple pueden distinguirse sistemas de manejo de turno o rotación (RFM-systems), sistemas de explotación esporádica (SPX systems) y sistemas de cubierta forestal continua (CCF systems), los cuales se esquematizan en la figura 1.

Los sistemas de manejo de turno o rotación con silvicultura estándar son comunes en muchas regiones del mundo. Los sistemas de explotación esporádica consisten en la extracción de los árboles más valiosos en intervalos de tiempo irregulares y se localizan comúnmente en áreas remotas con poco control público. Los sistemas de manejo de cubierta forestal continua se caracterizan por la realización de cortas selectivas y se aplican más frecuentemente en las regiones densamente pobladas y en algunos bosques tropicales.

Las plantaciones a gran escala comenzaron en Europa en el siglo XVIII. Durante la primera mitad del siglo XX los sistemas de rotación se establecieron en Estados Unidos, Australia, Nueva Zelanda, Sudáfrica, India, Chile, Indonesia y Brasil. Más tarde, en los años 50 Japón, Corea y China desarrollaron programas de reforestación masivos mediante plantaciones comerciales. Según el Global Forest Resource 


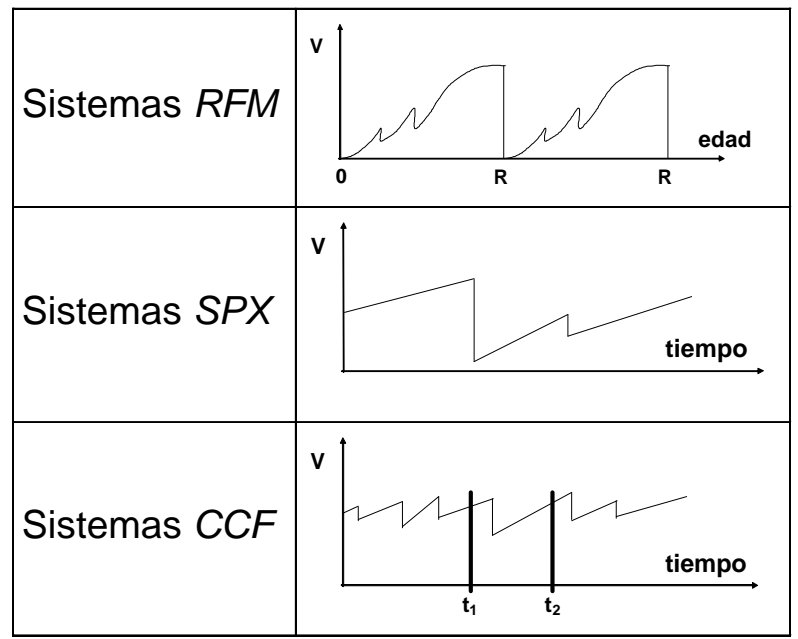

Figura 1. Representación esquemática del desarrollo de la biomasa (V) en función de la edad y del tiempo (ti) para tres diferentes sistemas de manejo forestal. RFM: sistemas de manejo de turno o rotación (R); SPX: sistemas de explotación esporádica; CCF: sistemas de cubierta forestal continua.

Assessment 2002, realizado por la FAO, la superficie de las plantaciones incrementó de cerca de 18 millones de hectáreas en 1980 a 187 millones de hectáreas en el año 2000.

Los estados situados en la zona Sur de los Estados Unidos cuentan con 11,6 millones de hectáreas de cuatro especies comerciales de pino: Pinus taeda, $P$. echinata, $P$. palustris y $P$. elliottii. Nueva Zelanda, Chile, Australia, España y Sudáfrica tienen establecidas 4,1 millones de hectáreas de Pinus radiata. Pinus patula y $P$. elliottii ocupan cerca de 1,3 millones de hectáreas en Sudáfrica, Argentina y Uruguay. Brasil cuenta con 400,000 hectáreas de Pinus caribaea y $P$. oocarpa. En 16 provincias en China las plantaciones de Cunninghamia lanceolata ocupan 8,75 millones de hectáreas. En Brasil, Australia, Zimbabwe y Malawi la superficie ocupada por plantaciones de Pinus elliottii, $P$. taeda y $P$. patula asciende a 1,7 millones de hectáreas (FAO, 2002).

Los sistemas de rotación se caracterizan por una estrategia de manejo bien definida con la finalidad de obtener el máximo beneficio económico. Los sistemas de explotación esporádicos se distinguen por el largo intervalo de tiempo entre operaciones de corta. La cosecha es a menudo drástica con la finalidad de extraer las existencias de más valor comercial. Algunos ecosistemas, como las sabanas en África con árboles valiosos o las taigas vírgenes pueden verse afectados por cortas esporádicas. Los sistemas de explotación esporádica se localizan en áreas remotas donde el control público es mínimo.

Los paisajes forestales son complejos sistemas abiertos con múltiples funciones. Comprender la dinámica natural de estos sistemas es un prerrequi- 
sito para el manejo. Consecuentemente, los métodos mecanicistas caracterizados por aplicar esquemas de tratamiento estandarizados, como los usuales en sistemas de manejo de bosques regulares, se están sustituyendo cada vez más por sistemas de manejo con orientación hacia procesos naturales. Manejo conforme a la naturaleza significa que se emplean especies adaptadas al sitio y que se aplican cortas selectivas; en Europa el manejo en cubierta forestal continua se está haciendo cada vez más popular, especialmente en los paisajes urbanos y suburbanos.

Los recursos forestales cumplen diversas funciones para la sociedad y consecuentemente debería ser condición que el manejo se realice sobre bases científicas, lo que constituye un difícil reto. Una tarea importante de la ciencia denominada "manejo forestal" es desarrollar herramientas prácticas para utilizar la experiencia acumulada en un paisaje científico muy fragmentado. Por esta razón es esencial que la investigación no se restrinja a resolver problemas específicos, sino que incluya también nuevos métodos para evaluar opciones de manejo y nuevas técnicas para el análisis y evaluación periódica de las prácticas forestales.

\section{OBTENCIÓN DE DATOS}

Como se ha comentado, los bosques son sistemas abiertos complejos con múltiples funciones. Para mantener la credibilidad, los responsables del manejo y los investigadores necesitan tomar observaciones en el campo. Dado que este es un proceso costoso, se requiere evaluar continuamente los métodos de inventario.

Los datos referidos al sitio constituyen una base esencial para la selección de las especies. El diagrama (Fig. 2) muestra un ejemplo de un sitio forestal en la provincia Cabo en Sudáfrica. El sitio es solamente adecuado de manera marginal para Pinus elliottii. Debido a la deficiencia de nutrientes, en plantaciones de $P$. radiata se recomendó emplear fertilizantes; $P$. pinaster alcanza tasas de crecimiento satisfactorias sin el empleo de fertilizantes; la cosecha se realiza sin problemas. El suelo está a menudo húmedo durante la estación lluviosa pero se seca rápidamente; la compactación no es un problema ya que se trata de un suelo de textura gruesa.

Las observaciones de parcelas permanentes representan una base de datos muy importante para desarrollar modelos de crecimiento. Durante un largo período se registran en las mismas los cambios cuantitativos y cualitativos de los atributos de los árboles. De esta manera las observaciones obtenidas permiten la construcción de un modelo de crecimiento para un conjunto de condiciones dado. Muchas tablas de producción se construyeron con base en datos de parcelas permanentes. Una desventaja del diseño de parcelas permanentes es el elevado costo de mantenimiento de la infraestructura de investigación y la larga espera por los datos.

Las parcelas temporales pueden proporcionar una rápida solución en situaciones donde no existen datos sobre el desarrollo forestal. Estas parcelas se miden sólo una vez pero cubren un amplio rango de edades y de sitios. La principal limitación de las parcelas temporales es el hecho de que no proporcionan información sobre tasas de crecimiento; por lo que no son muy recomendables. Se puede lograr un compromiso utilizando un sistema de parcelas de muestreo que mantenga las ventajas de las parcelas permanentes, como la obtención de tasas de crecimiento, y de las parcelas temporales, como la espera mínima por los datos. 


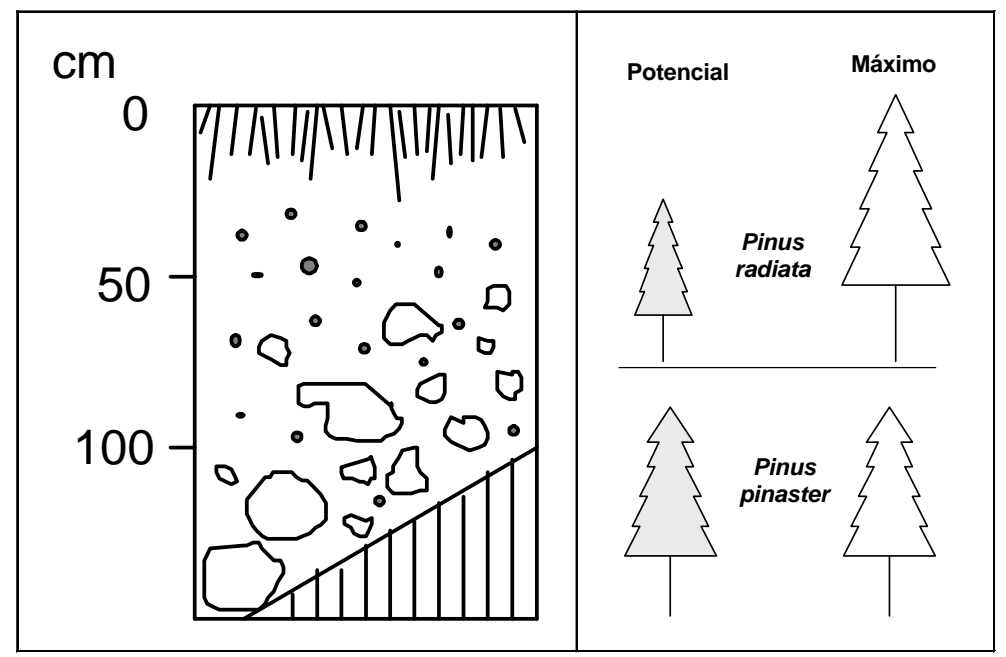

Figura 2. Ejemplo de un sitio en la provincia Cabo, Sudáfrica.

Las parcelas de intervalo se miden dos veces. El intervalo entre dos mediciones es un período de crecimiento sin perturbaciones. Las parcelas de intervalo se pueden establecer sobre un amplio rango de sitios, estados de desarrollo y tratamientos silvícolas. Estas parcelas combinan las ventajas de las parcelas permanentes y temporales. Se pueden obtener tasas de crecimiento para diferentes estados iniciales y tras una espera mínima se dispone de datos adecuados para modelar procesos.

\section{MANEJO FORESTAL SOBRE BASES CIENTÍFICAS}

En este capítulo se propone un concepto simple para el manejo forestal científico. Hoy en día existe el acuerdo generalizado de que el manejo forestal debe ser sustentable, basarse en resultados de investigación validados, aplicarse conforme a estándares ambientales aceptables y ser transparente al público. Estos objetivos se pueden alcanzar si: a) se evalúa una variedad de opciones forestales, b) se demuestra el manejo de manera comprensible en el campo y c) se registran y evalúan continuamente las actividades de manejo. Un sistema práctico para el manejo forestal científico que se base en estas premisas incluye tres elementos: Diseño de Opciones, Parcelas Demostrativas y Análisis de Eventos.

\section{Diseño de Opciones}

La teoría del diseño de opciones es simple: un paisaje arbolado cuenta con un determinado número de rodales, hay varias posibilidades de desarrollo para cada rodal y la tarea implica establecer las actividades de manejo a nivel de rodal. Actualmente existen numerosos ejemplos de aplicación de este concepto, sobre todo en el manejo comercial; siendo aplicables los trabajos de Ware y Clutter (1971); Hoganson y Rose (1984); Siitonen (1983); García (1990); Lappi (1992); Eid (1993); Peyron (1993); Rodríguez (1996); y Hoen (1996). Cuando se generan opciones de manejo (vías alternativas de desarrollo, escena- 
rios alternos) se deben realizar tres tareas: se requiere predecir el crecimiento de los árboles entre las intervenciones, se deben estimar daños ocurridos entre las mismas y finalmente se tiene que precisar el tipo y el peso de las intervenciones forestales. Para obtener esta información se deben utilizar algoritmos de extracción. Este modelo asume que un paisaje forestal es una agregación de rodales de diferentes tamaños y formas. Cada rodal se caracteriza por una población de árboles que posee un conjunto de atributos determinado. La figura 3 muestra un paisaje forestal hipotético con 3 rodales.

Planificar significa diseñar el desarrollo espacial del paisaje forestal a través del tiempo. Se considera la madera extraída y otros objetivos y restricciones tales como un cierto nivel de biodiversidad al final del período de planificación. El modelo de diseño de opciones forestales se basa en comprender que para cada parcela de terreno individual existen no sólo una, sino una variedad de opciones silvícolas disponibles, caracterizadas por una sucesión de actividades de manejo seguidas de un período de crecimiento natural. Cada opción tiene un valor específico. El modelo de opciones forestales ha sido definido por Torres y Brodie (1990) como se muestra en la figura 4.

La versatilidad del modelo radica en el hecho de que puede utilizarse para cualquier tipo de manejo forestal $y$ permite integrar experiencias de diferentes disciplinas. Para su aplicación se requiere predecir el crecimiento, los riesgos y las cortas futuras.

\section{cosecha hábitat}

\section{Período 1}
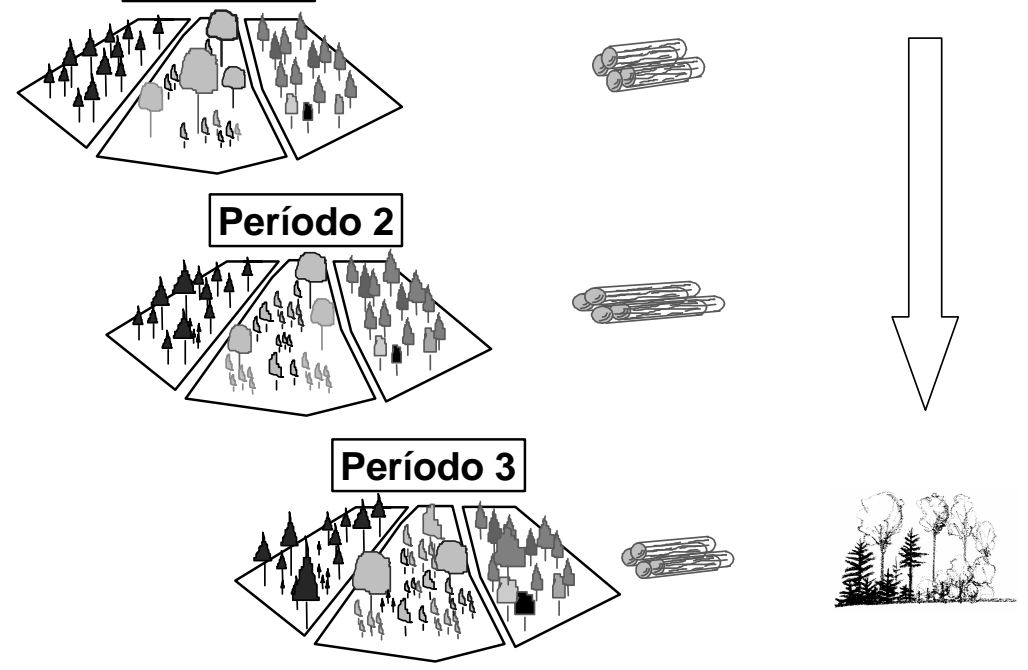

Figura 3. Modelo de múltiples opciones. Un paisaje forestal hipotético con 3 rodales. 


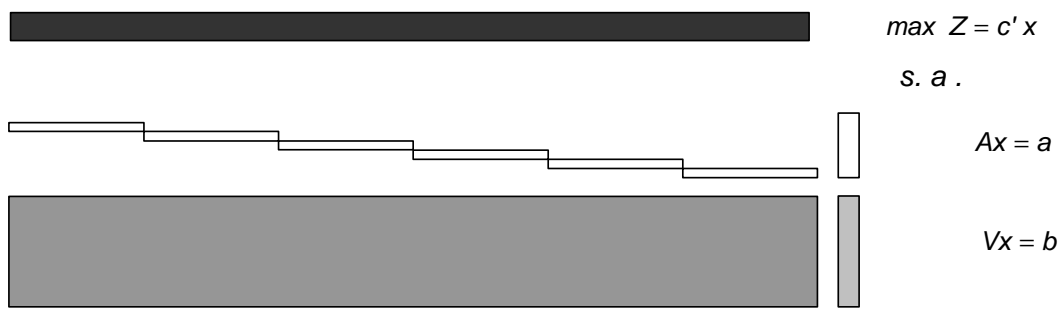

Figura 4. Representación gráfica del modelo de múltiples opciones.

c representa el vector de utilidades netas derivadas de cada opción de manejo forestal, $x$ es el vector de actividades forestales, A constituye la matriz de coeficientes técnicos que representa las superficies bajo cada opción de manejo y a es el vector de superficies de cada rodal. V es la matriz de volúmenes de cosecha bajo cada opción de manejo y b el vector de requerimientos de volumen en cada periodo de planeación.

Un ejemplo de un mapa dinámico con 41 rodales se presenta en la figura 5 . Los 3 mapas muestran diferentes tipos de aclareos en los diferentes rodales indicados por distintos tonos.

Modelos espaciales del paisaje como el anterior han sido utilizados por Chen y Gadow (2002) y Sánchez y Vilcko (2002). Chen utilizó Simulated Annealing combinado con ArcView. Sánchez y Vilcko emplearon el sistema de optimización lineal JLP desarrollado por Lappi (1992). El crecimiento y los tipos de aclareos fueron simulados a nivel de rodal empleando modelos de árboles individuales; la descripción del tipo de aclareo se realiza a nivel de rodal, pero el crecimiento se proyecta a nivel de árbol individual. El trabajo es complejo; requiere de habilidades cuantitativas y buenos modelos de crecimiento; un aspecto muy importante es la estimación precisa de la densidad máxima.

La predicción del crecimiento y de los riesgos, así como la evaluación de regímenes de tratamiento requieren de los conocimientos de diferentes áreas, pudiendo diferenciarse 3 grupos de disciplinas implicadas en investigación forestal: Ciencias Naturales (Clima- tología, Genética, Zoología, Edafología), Ciencias Sociales (Política Forestal, Economía) y Ciencias de la Ingeniería (Manejo Forestal, Silvicultura, Teledetección).

\section{Parcelas Demostrativas}

El segundo elemento del manejo forestal científico lo constituyen las denominadas parcelas demostrativas. La finalidad de una parcela demo es mostrar los diferentes tipos de silvicultura en el campo. Estas parcelas también se pueden emplear para la obtención de datos.

Las parcelas demo son particularmente útiles para formación y educación. Los efectos de un tratamiento silvícola específico se pueden hacer transparentes en el campo. Cada una de las disciplinas científicas puede participar en el análisis de las modificaciones de la densidad, del valor y la estructura forestal. El área basal extraída se puede utilizar para describir la intensidad de los aclareos, mientras que el tipo de aclareo se describe mediante la relación entre el número de árboles relativo extraído y el área basal relativa extraída. 


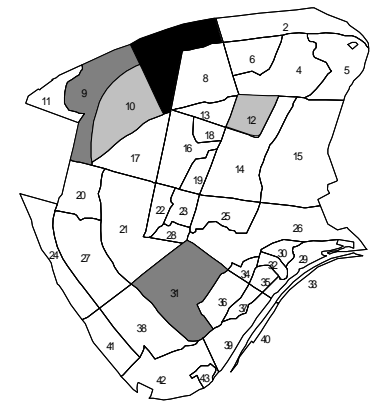

2005

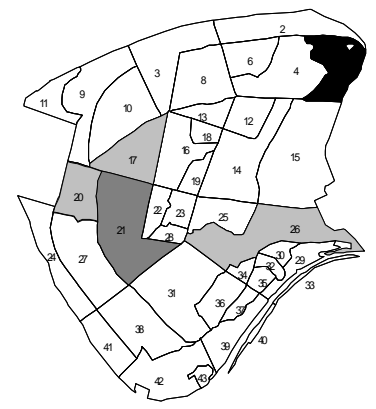

2010

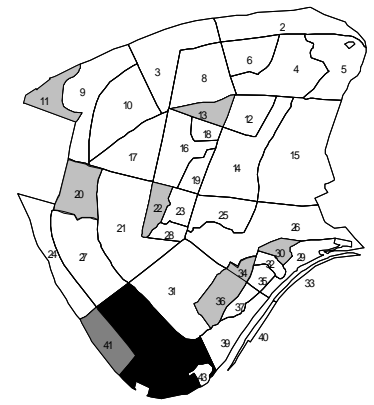

2015

Figura 5. Mapa de evolución de un paisaje forestal con 41 rodales. Los diferentes tipos de aclareos se indican en los rodales con de distintas tonalidades.

El cambio en la estructura espacial se describe a tres niveles: cambio del grado de agregación, cambio de la mezcla de especies y cambio en la diferenciación dimensional. Sería conveniente que las actividades de inventarización y registro periódico se pudieran combinar; este objetivo se puede alcanzar programando el inventario de manera que coincida con un tratamiento silvícola.

\section{Análisis del Evento Silvícola}

La dinámica del desarrollo de un paisaje forestal comprende una gran variedad de cambios como resultado de las actividades silvícolas. Un mecanismo efectivo para mantener el control sobre las actividades de manejo es el método denominado análisis del evento silvícola. La mayoría de los inventarios estiman las existencias residuales (que varían pronto debido a las operaciones de corta). El muestreo de eventos toma los datos de los rodales inmediatamente después de que los árboles hayan sido marcados pero antes del derribo, de tal manera que se dispone de información sobre: a) los árboles después del aclareo, b) árboles extraídos y c) árboles que permanecen después del aclareo.

El bosque se modifica por una corta y la modificación puede asumir muy diferentes formas. El análisis del evento silvícola clarifica estas modificaciones que pueden evaluarse en términos de cambio en la densidad forestal (extracción de biomasa), cambio en la estructura forestal (adecuación sitio-especie; distribución dimensional) y valor forestal (valor recreativo; calidad de fustes).

El desarrollo de los ecosistemas forestales es incierto por naturaleza; el análisis del evento silvícola facilita un manejo adaptativo que implica una evaluación cuantitativa regular de las actividades de manejo. El diagrama a la izquierda (Fig. 6) muestra un aclareo por lo bajo en un bosque de haya y a la derecha se esquematiza un aclareo por lo alto en el mismo bosque.

Los efectos en la distribución de la luz y la temperatura, la modificación de la estructura genética y el aspecto económico de las operaciones son bastante diferentes. La utilidad del método 

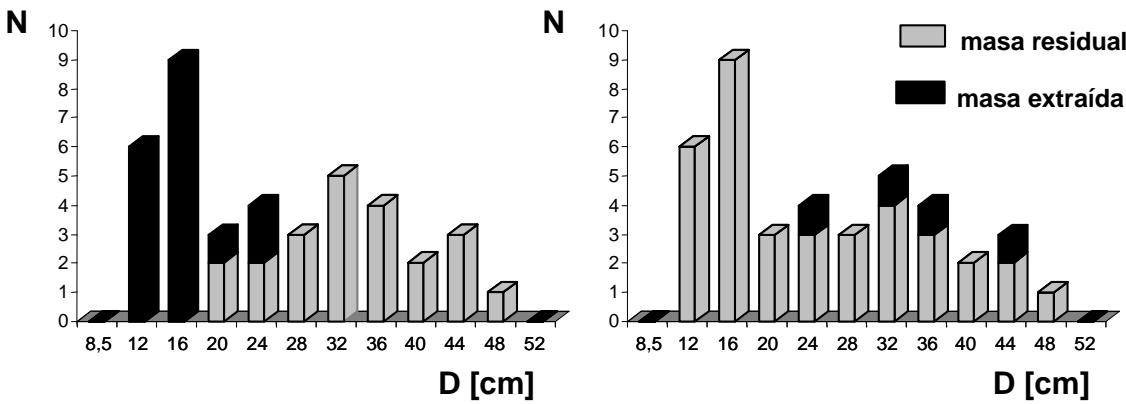

Figura 6. Efectos de las cortas en la masa forestal.

aumenta con la creciente incertidumbre en el manejo y con la complejidad en la toma de decisiones. Para ello el análisis del evento silvícola es específicamente apropiado en bosques irregulares y multiespecíficos manejados mediante el método de selección. La mayoría de los inventarios recogen las existencias (que pueden variar pronto como resultado de una operación de aclareo). Dedicando el mismo esfuerzo que para un inventario periódico, el inventario de la corta es capaz de producir simultáneamente información sobre los árboles antes del aclareo, los árboles extraídos y los árboles que permanecen en el rodal después del aclareo. Así, el inventario de la corta proporciona datos sobre cambios inducidos por el manejo que pueden ser utilizados para el análisis de eventos.

Durante varios años el análisis del evento de corta ha sido parte integral del programa académico de la Facultad de Ciencias Forestales en Göttingen, Alemania. Un análisis básico implica las modificaciones de la estructura forestal y del valor de las existencias. Este análisis se complementa con una evaluación de los efectos de la corta sobre las condi- ciones del suelo, la estructura genética y el clima forestal integrando todas las disciplinas en el análisis. El mapa muestra la ciudad de Göttingen situada dentro de un típico paisaje forestal urbano (Fig. 7).

La ciudad está rodeada por bosques y el público quiere saber cómo se manejan las áreas arboladas. El análisis del evento silvícola es un método efectivo para monitorizar el manejo forestal sostenible.

\section{CONCLUSIONES}

Los límites de la ciencia están marcados por la imposibilidad de predecir de manera absoluta sistemas físicos y biológicos. Sin embargo, los conocimientos científicos son la base para la toma de decisiones racionales. Un reto para el manejo de bosques es la sustitución de doctrinas silvícolas que no han sido examinadas, por conocimientos científicamente comprobables. Este objetivo se alcanza a través del análisis sistemático de las variantes de desarrollo y de las operaciones de corta. 


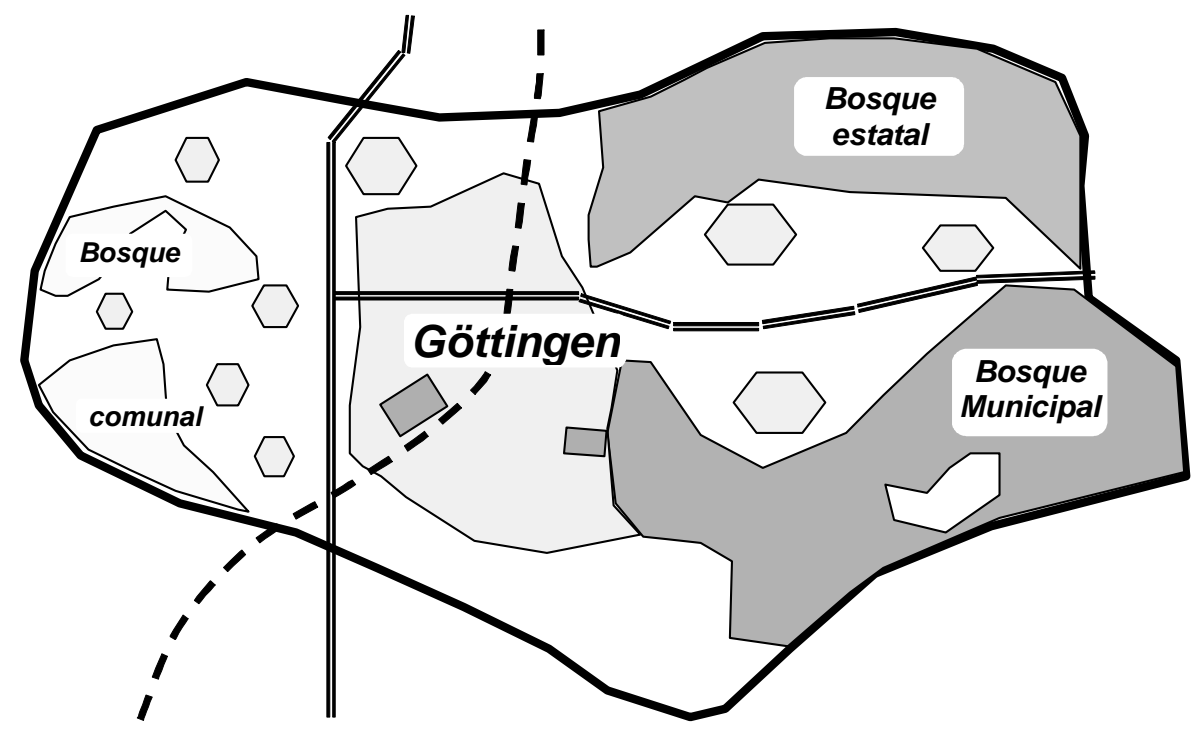

Figura 7. La ciudad de Göttingen, Alemania, y alrededores; un ejemplo de un paisaje forestal urbano en Europa.

En conclusión, se puede observar que el manejo forestal con bases científicas reconoce ecosistemas forestales $y$ necesidades económicas, emplea metodologías adecuadas de obtención de datos y desarrolla estrategias efectivas para la planificación y análisis, como las descritas en este trabajo: diseño de opciones forestales, demostración del manejo, análisis del evento silvícola.Los bosques representan una reserva renovable de materias primas para la industria de la madera, un recurso natural con amenaza de desaparición en regiones empobrecidas y un hábitat virgen de elevado valor recreativo y espiritual en las sociedades densamente pobladas. La implementación exitosa de sistemas de manejo forestal sustentable requiere objetivos operacionales y un concepto de manejo práctico con métodos adecuados para la estimación de los recursos, la planificación y el control. El desarrollo forestal es incierto por naturaleza y el manejo tiene que ser adaptativo. La utilización de un modelo de desarrollo forestal realista para la generación de opciones alternativas de tratamiento silvícola facilita este tipo de manejo. El modelo incluye tanto el crecimiento natural como los riesgos y los aclareos intermedios. El análisis del evento silvícola también facilita el manejo adaptativo.

\section{REFERENCIAS}

Abdul R., M.A. y S. Appanah. 2000. Forest resources management in South-East Asia - new directions. Proc. XXI IUFRO World Congress Vol 1:933-943.

Beer, J.; M. Ibrahim y A. Schlönvoigt. 2000. Timber production in tropical agroforestry systems of Central 
America. Proc. XXI IUFRO World Congress Vol 1:777-786.

Bousquet, F.; C. Le Page; M. Antona y P. Guizol. 2000. Ecological scales and use rights - the use of multiagent systems. Proc. XXI IUFRO world congress Vol 1:730-742.

Buongiorno, J y J.K. Gilles. 2002. Decision methods for forest resource management.. Macmillan, Nueva York. 439 p.

Cancino, J. y K. v. Gadow. 2001. Stem number guide curves for unevenaged forests - development and limitations. In: Gadow, K. v.; J. Nagel y J. Saborowski. 2001. Continuous Cover Forestry - Assessment, Analysis, Scenarios. Managing Forest Ecosystems Vol. 3. Kluwer Academic Publishers. Dodrecht. p:279-288.

Chen, B.W. y K. v. Gadow. 2002. Timber harvest planning with spatial objectives. Forstwiss. Cbl. 121:25-34.

Dada, S.A. 2000. Sustainable forest management in Africa - the challenges and issues at stake. Proc. XXI IUFRO world congress Vol 1:945-951.

De Camino, R. 2000. Regional scenarios in management of natural resources - Latin America. Proc. XXI IUFRO world congress Vol 1:944.

Eid, T., 1993. Models for economical forest management planning in Norway. Proc. Symp. Modelling in forest management planning and managerial economics - a critical investigation. Lithuanian Agricultural Academy:35-43.

Emborg, J. y J.B. Larsen. 1999. How to develop plantations into forests in order to achieve stability and func- tional flexibility - a North European perspective. Proc. International experts meeting on the role of planted forests for sustainable forest management. Santiago, Chile 6-9 abril. p:135-147.

FAO (Organización de las Naciones Unidas para la agricultura y la alimentación). 2001. Situación de los bosques en el mundo 2001. Roma. $174 \mathrm{p}$.

FAO (Organización de las Naciones Unidas para la agricultura y la alimentación). 2002. State of the World's Forests. (también disponible en http://www.fao.org/forestry/fo/ sofo/sofo-e.stm)

Folch, R. y J.M. Camarasa. 2000. Encyclopedia of the Biosphere. Vol. 1-11; Gale Group.

Gadow K. v. y J. Pummalainen. 2000. Scenario planning for sustainable forest management. In: Gadow, K v., T. Pukkala y M. Tomé (eds). Sustainable forest management. Managing Forest Ecosystems Vol. 1. Kluwer Academic Publishers. Dodrecht. p:319-356.

García, O., 1990. Linear programming and related approaches in forest planning. N. Zealand J. of For. Science 20(3):307-331.

Garfitt, J.E. 1995. Natural management of woods: Continuous cover forestry. John Wiley \& Sons Inc. Nueva York. $152 \mathrm{p}$.

Hardin, G., 1968. The tragedy of the commons. Science 162:1243-1248.

Harvey, C.A., C.F. Guindon; W.A. Haber; D. Hamilton de Rosier y K.G. Murria. 2000. The importance of forest patches, isolated trees and agricul- 
tural windbreaks for local and regional biodiversity, - the case of Monteverde, Costa Rica. Proc. XXI IUFRO world congress Vol 1:787798.

Hoen, H. 1996. Forestry Scenario Modelling for Economic Analysis experiences using the GAYA-JLP model. In: Päivinen, R.; L. Roihuvuo. y M. Siitonen (eds). 1996. Largescale forestry scenario models experiences and requirements. European Forest Institute. EFI Proceedings No. 5:79-88.

Hoganson, H.M. y D.W. Rose. 1984. A simulation approach for optimal timber management scheduling. For. Sci. 30 (1):200-238.

Lappi, J. 1992. JLP - a linear programming package for management planning. The Finnish Forest Res. Inst., Res. Paper 414:131 p.

Meyer, W.H. 1930. Diameter distribution series in even-aged forest stands. School of Forestry, Yale Univ. Bull. 28:105 p.

Nik M., M.; E. Varques B. y A.A. Mohamad. 2000. Role of forestry in landscape rehabilitation - Malaysian experience. Proc. XXI IUFRO world congress Vol 1:297-309.

Pearce, D.W. y R.K., Turner. 1990. Economics of natural resources and the environment. John Hopkins University Press, Baltimore. 378 p.

Peyron, J.L. 1993. Présentation illustreé d'une méthode de planification de la gestion forestière et de détermina- tion de l'effort de régénération. Rev. For. Fr. XLV (1):59-67.

Rodriguez, L. 1996. A Microcomputer Program for Solving Forest Scheduling Problems with Heuristic Approaches. In: Päivinen, R.; L. Roihuvuo y M. Siitonen (eds). 1996. Large-scale forestry scenario models - experiences and requirements. European Forest Institute. EFI Proceedings No. 5:153-166.

Sánchez Orois, S. y F. Vilcko. 2002. Bewertung und Optimierung von Vornutzungen in Fichtenbeständen. Forstwissenschaftliches Centralblatt 121:250-264.

Siitonen, M. 1983. A long term forestry planning system based on data from the Finnish national forest inventory. Univ. of Helsinki, Dept of For. Mens. \& Mgmt Res. Note 17:195-207.

Thomasius, H. y P.A. Schmidt. 1996. Wald, Forstwirtschaft und Umwelt. Economica Verlag. Bonn. 433 p.

Torres R., J.M. y J.D. Brodie. 1990. Adjacency constraints in harvest scheduling. Can. J. of For. Res. 20:978-986.

Vanclay, J.K.; R. Muetzelfeldt; M. Haggith y F. Bousquet. 2000. Flores helping people to realize sustainable futures. Proc. XXI IUFRO world congress Vol 1:723-729.

Ware, G.O. y J.L., Clutter. 1971. A mathematical programming system for the management of industrial forests. For. Sci. 17:428-445. $\diamond$ 